\title{
1 Integrating RC bridge defect information into BIM models
}

2 Philipp Hüthwohl ${ }^{1}$, Ioannis Brilakis ${ }^{2}$, André Borrmann ${ }^{3}$, Rafael Sacks ${ }^{4}$

$3{ }^{1}$ Ph.D. Candidate, Dept. of Engineering, Univ. of Cambridge, Trumpington St., Cambridge CB2 1PZ,

4 U.K. (corresponding author). E-mail: ph463@cam.ac.uk

$5 \quad$ 2Laing O’Rourke Reader, Dept. of Engineering, Univ. of Cambridge, Trumpington St., Cambridge

$6 \quad$ CB2 1PZ, U.K. E-mail: ib340@cam.ac.uk

$7 \quad{ }^{3}$ Prof. Dr.-Ing., Chair of Computational Modeling and Simulation, Leonhard Obermeyer Center,

8 Technische Univ. München, Arcisstraße 21, 80290 Munich, Germany. E-mail: andre.borrmann@tum.de

$9{ }^{4}$ Associate Professor, Faculty of Civil and Environmental Engineering, Technion - Israel Institute of

10 Technology, Haifa 32000, Israel. E-mail: cvsacks@technion.ac.il

\section{ABSTRACT}

12 Reinforced Concrete bridges are a vitally important part of our infrastructure. The status of this

13 infrastructure needs to be monitored on a continuous basis in order to ensure its safety and

14 functionality. This is currently being done by authorities worldwide via bridge inspection reports. The

15 format and storage of these reports varies considerably across different authorities around the world

16 and is sometimes comprised into a bridge management system (BMS). The lack of standardization

17 severely hinders the use of inspection information for knowledge generation use cases of both

18 practitioners and researchers. This paper presents an exploratory analysis and as a result an

19 information model and a candidate binding to IFC to categorize inspection information of RC bridges

20 and to standardize storage of this information in a format that is suitable for sharing and comparing it

21 between different users and varying requirements. We were able to show in three steps, that IFC in its

22 latest version IFC 4 provides sufficient functionality to serve as a basis for integrating relevant defect

23 information and imagery. Firstly, we extracted types of defects and properties needed for bridge 
24 assessment from existing bridge inspection manuals. Secondly, we modelled the defect entities, their

25 properties and relations and thirdly, mapped them to appropriate IFC entities. A prototypical

26 implementation serves as a proof of concept for automated sharing and comparing of information

27 needed in RC bridge inspections and for establishing a knowledge base for bridge performance over

28 time and across authorities.

\section{INTRODUCTION}

30 Bridge inspection data is collected through intense human effort. The resulting bridge condition

31 information is kept in bridge inspection reports and is used for maintenance and preparation of

32 subsequent inspections. These reports are stored in diverse and non-interoperable BMS systems or

33 even on paper. This restricts their use to the intended workflows only (Johnson et al. 2016; The

34 Highways Agency 2007), which has a threefold impact: First, for industry, having a closed and

35 proprietary format precludes inspectors from exchanging bridge condition information easily for

36 either educational use or for getting an expert opinion from someone not within the same authority.

37 Secondly, this mode of record-keeping makes mining the data an extremely challenging task, which

38 can hinder the development of new methods for designing, building and maintaining bridges in the

39 future (Dekker 2006). Thirdly, it prevents using the information for structural and other analysis

40 software for use in bridge repair and retrofit which forces further survey and wasting money through

41 rework. This research explores how to overcome this.

42 The quality of inspection data, as it exists today, varies greatly with much being of poor quality

43 (Phares et al. 2004). Maintenance decisions are made based on this data and more than $£ 18$ billion per

44 year are spent for bridge maintenance in the US and United Kingdom alone (ASCE 2017; Department

45 for Transport and Highways Agency 2014). Kong et al. (2003) claimed that preventive maintenance

46 decisions can reduce the maintenance cost in infrastructure by up to $65 \%$, or $£ 11.7$ billion, but require

47 precise knowledge about the bridge condition.

48 Inspectors typically list all defects identified during a bridge inspection in a bridge inspection report.

49 They are required to describe affected elements, type of defects and their severities. For instance, 
50 Table 1 shows relevant defects for reinforced concrete (RC) along with their appearance and

51 interpretation criteria for selecting the most appropriate condition rating. Lines correspond to the

52 different defect types, columns correspond to the respective condition ratings. Inspectors can also

53 comment on specific aspects of a defect, such as how they inferred the condition rating of a defect,

54 recommend a type of maintenance needed and estimate its cost.

55 A bridge inspection report is either printed on paper or stored as an electronic copy in a Bridge

56 Management System (BMS). A BMS allows storage, manipulation and management of bridge data

57 and supports engineering processes, asset management and resource planning. On-site data can be

58 collected using electronic handheld devices. Electronic inspection forms consist of dropdown fields

59 that enable inspectors to quickly input data and to reduce input errors. A bridge inspection report

60 generally gives a holistic view of a bridge condition at a specific point in time, yet is laborious to

61 create. An inspector spends more time on actually writing a bridge inspection report than on

62 inspecting the bridge on site (Kouridi and Brilakis 2016).

63 Such reports also often lack in quality and completeness (Moore et al. 2001). As a result, inspectors

64 and engineers have difficulty understanding the deterioration over time of a specific defect element or

65 defect position; they have to read through all existing reports searching for comments on a specific

66 element or position. This process is laborious and error-prone, especially when taking the number of

67 biannual inspections into account over an average bridge lifetime of more than 40 years (The

68 Highways Agency 2007; U.S. Department of Transportation 2012). Inspectors cannot filter and easily

69 find previous comments and defect findings based on position, element type, time or severity.

70 Recordings of a single defect identified in several bridge inspection reports are not logically linked

71 such that an inspector can easily access old records.

72 There is still no established standard between different DoTs and companies (Johnson et al. 2016; The

73 Highways Agency 2007), even though BMSs are developed to support an objective decision process

74 based on inspection data. Non-compatible bespoke formats for storing bridge defect information exist.

75 Inspection reports usually remain in a company's BMS which makes sharing them with anyone

76 outside the organization, for example bridge consultants or contractors, difficult and error prone. This 
77 is a classic problem of information islands. Furthermore, BMSs are managed on the level of the whole

78 structure and of nominal bridge elements; they do not store or represent bridge or defect geometry.

79 This means information cannot be assigned to a specific part or location of an element, only to the

80 element ID. The only way to record defect shape and location is through a freehand sketch or a

81 photograph. Hence, understanding the extent of a defect or comparing the extent to a prior report

82 entry is difficult.

83 Bridge inspection guidelines define the operational process of an inspection. They are used for

84 inspector training and also serve as reference book during an inspection. Each authority publishes

85 individual inspection guidelines adjusted to their particular needs. Differences originate from the geo-

86 location and thereby conditioned properties such as weather, distance to an ocean or risk of

87 earthquakes. Inspection guidelines describe what and how to generally document bridge defects.

88 According to Highways Agency, an inspector has to collect "relevant data and describing defects in

89 terms of their type, location, extent, severity and, if possible, cause" (The Highways Agency 2007). It

90 also provides material-type specific tables for different defects and a description of how to determine

91 a condition rating, as Table 1 is for reinforced concrete.

92 The problem is that this procedure is vague and leaves room for manual interpretation, for example on

93 how to measure a specific property. Inspectors are expected to distinguish crack severities by

94 measuring crack width with a crack gauge. But what exactly has to be measured? The maximum or an

95 average? Likewise, for thaumasite, distinction between different condition levels is even more vague;

96 guidelines refer to minor, moderate and major thaumasite without defining minor, moderate and

97 major. Inconsistent measurements lead to unreliable condition ratings and difficulty in clearly

98 understanding if a defect dimension has increased over time. Phares et al. identified $56 \%$ of average

99 condition ratings being incorrect with a $95 \%$ probability and inspection notes concerning important

100 structural defects show significant variability and many times are completely omitted (Phares et al.

101 2004). Cracks, for example, could theoretically shrink in width if a different location is used for

102 measurement. To the authors' knowledge, no explicit and comprehensive measurement standards 
103 exist for defect properties when conducting a condition assessment; both in terms of what to measure

104 and how to measure.

105 This paper presents exploratory research meant to derive a useful information model for integrating

106 bridge defect information into a BIM model in the end, rather than causal research meant to derive a

107 new process. First, relevant information is identified by analysing established inspection guidelines

108 from four authorities (Alberta Infrastucture and Transportation 2008; Johnson et al. 2016; Queensland

109 Department of Main Roads 2004; The Highways Agency 2007) and the SeeBridge IDM (Sacks et al.

110 2016), which is based on the Israel bridge inspection guidelines and the European bridge inspection

111 guidelines being developed. We compare defect types and extract their required properties for

112 condition assessment. This list of relevant defect-type dependent properties is then further refined to a

113 hierarchical structure of hierarchically modelled defect information objects, which is then matched to

114 IFC entities for integrating defect information into the BIM model. Finally, a prototype

115 implementation serves as a demonstration of utility and, as the presented results fully comply with

116 IFC, an analysis shows how much of the applied IFC standard is supported by existing IFC viewers.

\section{BACKGROUND}

118 Two questions arise for integrating defect information: which information is relevant and how can it

119 be modelled?

120 There is no comprehensive and justified definition of which defect properties are necessary for

121 condition assessment and maintenance processes. However, there are related fields where these

122 definitions exist: The Federal Highway Administration (FHWA) has released a detailed guide for

123 pavement inspection on how to inspect and document defects depending on the pavement material

124 type (Federal Highway Administration 2003). For example, seven different crack types are explained

125 for asphalt concrete surfaces along with a sketch indicating location and pattern for each defect type

126 and also condition state. In addition, measurements are defined for each condition state along with a

127 description of how and where to take measurements. Similar definitions are published by other

128 authorities (Minnesota Department of Transportation 2003; Wallis 2007). Defect types relevant for 
129 bridge inspection are known. What is missing, however, is a complete definition of defect-type

130 dependent and general properties which leave no room for interpretation to an inspector (incl.

131 definition of measuring quantity, unit, location, tools), plus a condition rating standard that is solely

132 based on these objective measurements.

133 The subsequent question is how to model relevant defect information. BMSs are most commonly used

134 to manage bridge defect information as a part of the general bridge stock information. Each bridge is

135 represented by a dataset where documents can be added, such as bridge inspection reports, or

136 attachments, such as images taken during an inspection or design drawings. Detection methods for

137 detecting defects, such as cracks, often use images to store defect maps (Adhikari et al. 2014; Zhu et

138 al. 2011). These defect maps are equal in size as an input image. The difference is that each pixel

139 colour value indicates an undamaged or damaged class assignment at a specific position as visualized

140 in Figure 1. Besides a binary representation, it can also be used to represent a pixel-based

141 classification result for multiple classes in one image, each class being represented by a pre-defined

142 colour. This process of semantically segmenting is used in many different fields, for example for

143 images taken with a car camera and segmenting them into classes such as roads, cars and signs

144 (Badrinarayanan et al. 2015). Defect maps are designed for representing a segmented classification

145 result to an input image on a pixel level, not a class level. Hence, clusters of pixels have to be

146 separated based on their colour and location.

147 Lee et al. used the Drawing Exchange Format (DXF) file format commonly used in Computer Aided

148 Design (CAD) to make defect information accessible for bridge management systems (Lee et al.

149 2008). This format was originally defined and used for storing and exchanging technical drawings

150 during the design and construction phase of an industrial product. The advantage of DXF is that it is

151 able to represent geometric information using vectors instead of locally separated pixels in images.

152 Defects can actually be represented as a polygon instead of a number of pixels. This means that it is a

153 machine-readable format that can be used directly for analysis. However, this schema for defect

154 geometry is separate from the BMS itself, and defect data cannot be carried integrally with geometry

155 in a DXF file. Both file formats were not intended for storing inspection data and crucial parts are 
156 missing for the storage of bridge defect information, most notably the corresponding element, the

157 exact position and orientation on the element and the size of the surface patch covered by the

158 image/design file.

159 Building Information Modelling (BIM) is a process intended to electronically model the whole

160 lifecycle of a building in all its aspects, including its 3D geometry. Mainly used for planning and

161 construction, it was originally designed to also share data and knowledge resources to support

162 management, utilization, renovation, and demolition activities (Eastman et al. 2011). While BIM is a

163 data and process description, many companies have developed their own proprietary data

164 representations of BIM models. One of the main limitations is that they are not exchangeable between

165 different platforms and are proprietary, which means that they are not freely accessible and subject to

166 changes by their manufacturers.

167 Mirzaei et al. examined, if Autodesk Revit can be used for bridge inspection information (Mirzaei et

168 al. 2012). They identified limited support for inspection specific features such as attaching images to a

169 specific element. McGuire et al. compared common BIM platforms (LEAP Bridge, Tekla Structures,

170 Revit) for modelling defects followed by structural performance testing. Their work focuses on the

171 structural assessment and the corresponding defect modelling requirements (McGuire et al. 2016).

172 Integrating or modelling defect information in conformity with existing inspection and condition

173 assessment guidelines was not part of their scope. As a result, the integrated defect properties (based

174 on damage cubes only for representing the damaged area) differ from the ones that are required by the

175 inspection manuals (e.g. width of cracks, exposed reinforcement for spalls). Hence, using this method

176 for bridge inspection in general will fail validation because essential inspection guideline

177 requirements are missing. For this reason, an information model for bridge inspection needs to start

178 with analysing existing inspection guidelines. Borrman et al. presented a system for building

179 condition rating based on 3D bridge models. It allows marking defects at surfaces of individual bridge

180 components. For importing geometry information, the proprietary ACIS file format SAT has been

181 employed (Borrmann et al. 2012). 
182 Industry Foundation Classes (IFC) define an interchangeable open data model for BIM that is

183 supported by most commercial software systems and can overcome restrictions between proprietary

184 platforms. IFC version $2 \times 3$ was released in February 2006 and is the most commonly used IFC

185 standard. It can facilitate maintenance information and as-is performance data, such as current

186 condition or damage states for facility management (William East et al. 2013). Anil et al. used IFC to

187 model damage information for post-earthquake assessment of reinforced concrete frames (Anil et al.

188 2013; Ma et al. 2015). More specifically, defect maps were added using

189 IfcGeometricRepresentationSubContext. Extracted crack properties were linked as external text files

190 using IfcRelAssociatedDocument, and IfcSurfaceStyleWithTextures was proposed to link

191 corresponding external crack images. This work shows, how to use the abstract data model of IFC to

192 model defect data. The presented work, however, is not sufficient for defect documentation in a

193 bridge inspection, as it was designed solely for cracks during an earthquake inspection and therefore

194 does not define which defect class dependent property sets for bridge inspection exist and how to map

195 defect texture onto a specific element surface.

196 BIM and IFC were developed with a focus on buildings. Horizontal infrastructure projects have

197 unique requirements such as alignment. To address this, the responsible instance for enhancing the

198 IFC standard, buildingSMART International Ltd., started a new committee, the Infrastructure Room.

199 This committee is working on infrastructure-specific standardization projects for rail, road, bridge and

200 tunnel (buildingSMART International Ltd. 2017a). Bridge information modelling (BrIM) was given

201 the highest priority because they are relatively close to buildings, considerable work has been done in

202 France and most existing bridge infrastructure is close to reaching its designed life span. Committee

203 work to date has not yet taken bridge inspection requirements into account (Hartmann and Director

204 2016).

205 Tanaka et al. proposed an IFC extension based on a more recent version of the IFC standard (IFC4)

206 and the Bridge Information Modelling (BrIM) standard. They introduced new IFC entities for

207 documenting inspected regions (IfcMeasuredRegion) and defect findings (IfcDegredation,

208 IfcDegredationElements) along with new connection types to connect instances of the new entities 
209 with bridge elements (IfcRelConnectsToMeasuredRegion) and to connect defect findings at different

210 timestamps (IfcRelConnectsToTimeVariations) (Tanaka et al. 2016). Crucial parts for holistically

211 modelling defect information are missing from Tanaka et al.'s extension: First, it did not investigate

212 what data is relevant for condition assessment. Secondly, it did not explain why the existing IFC

213 standard, already an extensive standard, does not provide sufficient entities and connections to meet

214 the requirements for bridge defect information. To date, no-one has assessed whether IFC4 can be

215 used for adding bridge defect information without extending the standard.

216 Existing work for the stated problem can be summarized as follows: Bridge inspection guidelines

217 define the way an inspection is performed, the relevant defect types and a documentation schema.

218 Each authority publishes its own inspection guidelines, which vary considerably, and maintains its

219 own BMS.

220 In conclusion, based on the state of research, although methods for managing bridge defect

221 information exist, essential gaps in knowledge remain: (1) There is no comprehensive and justified

222 definition of what defect (classes/types/properties or mix of them) is scientifically necessary for

223 bridge condition assessment and maintenance processes. (2) There is no IFC compliant schema or

224 data extension model that is able to store the defect (classes/types/properties) effectively for condition

225 assessment and maintenance purposes. (3) It is unknown whether the existing IFC schema richness is

226 sufficient to hold defect (class/type/property) data as explained above.

227 The objective of this work is to address the gaps in knowledge by answering the following research

228 questions:

229 - What are the transnational bridge inspection guideline requirements regarding documentation of

230 inspections in general and of the relevant defect types and defect-type dependent properties?

231 - What building information modelling data structures are appropriate for representation of the

232 consolidated documentation requirements?

233 - How can the proposed information modelling structures be bound into an existing BIM schema 234 definition? 
236 The research was exploratory in nature, and followed the methodology framework depicted in Figure

237 2. We analysed multiple transnational and transcontinental bridge inspection guidelines regarding

238 their general inspection information and regarding relevant RC defect types, to comprehensively

239 cover global inspection requirements instead of focusing on one guideline only. For the defect types,

240 we then extracted the raw properties usually accessible using the condition rating tables. This is the

241 information which later on lets an engineer interpret and assess the findings. We consolidated the

242 properties by identifying and removing / splitting overlap to avoid redundancy. We compiled both the

243 general information and the defect information in a hierarchical structure. Separate data fields only

244 represent raw data; a hierarchical structure converts it to meaningful information. We then matched

245 this structure to an existing BIM standard schema to make it applicable for the existing bridge

246 inspection process.

\section{INSPECTION REQUIREMENTS}

248 Inspection guidelines have common practices, but, as mentioned before, authorities adapt them to

249 their specific needs. For example, if the geolocation of an authority's area has no cold weather, it will

250 not list defects induced by freeze thaw cycles. We have compared multiple inspection guidelines in

251 order to extract representative properties for this work. We chose inspection guidelines in a way to

252 cover a variety of continents, climate zones and tectonic regions. We were limited to the ones that

253 were available in English. A list of the peer reviewed documents can be found in Table 2. In general,

254 many US inspection guidelines, including the one from California, are based on a reference inspection

255 guideline published by American Association of State Highway and Transportation Officials

256 (AASHTO) and therefore are related (AASHTO 2013). Inspection guidelines were compared

257 regarding their general information, the reinforced concrete defects, severities and severity distinction

258 features. Other documentation blocks, such as maintenance requirements, were not considered.

259 An inspector provides general information about a conducted inspection that are essential for the

260 correct assessment of a bridge and hence can be considered as background information to the bridge 
261 damage information. This general information typically consists of three blocks. A first block is

262 information that helps assigning a bridge inspection report to a specific bridge and gives background

263 information about the structure type. It consists of a structure ID, bridge name, street name,

264 construction type, bridge age and material. A second block documents additional information

265 regarding the inspection itself. This is the date, time, duration and type of inspection, weather

266 condition and if additional tools were used during the inspection. A final block lists responsible

267 people such as the chief inspector or assistant. All the inspection guidelines reviewed require such

268 information. The UK guideline has the most detailed general information block with additional fields

269 for completeness and photographs taken.

270 Inspection guidelines define multiple defect types and their relevant properties. An inspector is

271 expected to generally collect all relevant information describing defects in terms of their type,

272 location, extent, severity and, if possible, cause. Inspectors should give a clear and accurate

273 description of the condition of a structure. We compared five inspection guidelines regarding the

274 listed defect types for reinforced concrete and how condition states are distinguished. Defects on pre-

275 stressed reinforced concrete and construction defects were not considered for simplicity. It should also

276 be noted, that we did not try to validate the correctness or completeness of the defect description, but

277 rather more to identify and extract a representative list of defects and their measurable defect

278 properties. The resulting summary is vague and possibly not sufficiently precise for a structural

279 engineer since the existing inspection documents are already vaguely formulated. It is not part of this

280 work to formulate better guidelines, but to extract defect properties from existing inspection

281 guidelines. These properties were either taken from the guidelines defect description or from the

282 corresponding condition ratings. We compared descriptions for condition ratings and extracted

283 properties of the most specific ones. For example, quantitative descriptions with definite

284 measurements such as width, length and height were preferred to general qualitative descriptions such

285 as slight, minor and major. Resulting defects and their type dependent properties are listed in Table 3.

286 Defects, that were only present in one inspection guideline, are not further investigated but are listed

287 in the column "other defects". 
- Cracks - All inspection guidelines list cracks as a defect class. Only the Israel bridge inspection guideline differentiates crack types based on their likeliness of affecting the stability of an element or structure, which already requires an assessment. It also gives distinct minimal and maximal width values for corresponding condition ratings. The UK guideline explains different crack causes: flexural cracks, shear cracks and torsion cracks, but they are all documented as a single crack type with different severity. One needs to know if the crack occurs in an area of high flexural behaviour, if it is located close to the supports, and how it is oriented relative to the supports in order to distinguish them. Hence, the properties that have to be extracted are width of a crack, the flexural behaviour in this area, if it is close to a midspan or support and the orientation relative to a support. A definition of high flexural behaviour or how to interpret distance to a support is missing.

- Delamination - Delamination conditions are distinguished by early signs of delamination (cracks along with rust staining in the UK, crack width in Israel) and by low or high flexural and/or shear action in an occurring area. A severe delamination state also takes exposed reinforcement into account. Delamination is a defect that can be visible in multiple ways, it might even be not visible at all. Assessing delamination requires to detect basic defects cracks and rust staining. In addition, it requires knowledge about the location and the flexural and shear behaviour in this area.

- Spalling / Exposed Rebar / Corrosion - Spalling, exposed rebar and corrosion is grouped as they cannot occur separately. Exposed rebar is accompanied by spalling, and corrosion refers to the corrosion of its reinforcement steel bars. The UK inspection guideline requires an inspector to identify if a spall is minor or major, if and what type of reinforcement bars are exposed (shear links / main bars) and to what state the exposed rebar is corroded (general/pitting). There is no additional information given on how to distinguish between minor and major spalls. The guideline of California and Alberta provide a better understanding by taking the defect diameter and/or depth into account. The relevant properties are diameter, depth, exposed rebar and corrosion state.

- Efflorescence - Efflorescence is visible as white deposits on a concrete surface. This defect is listed in Alberta, Israel and California. No additional definition of which properties are relevant for this 

type of defect is given. More important is to recognize which other defect types come along with

316 this defect, for example, if cracking or spalling occurs. The Israeli inspection guideline points out, 317 that these other defects have to be assessed separately. Hence, the properties that are required for 318 Efflorescence are if accompanied by other damages and if this other damage is structurally 319 relevant.

- Freeze-thaw - This defect is listed by the UK and Israel inspection guideline. However, both fall short of giving a distinct definition on how to identify and distinguish condition states of freezethaw. Both state slight, minor, major freeze-thaw as condition state without giving additional explanation. Only the Israel guideline lists an additional feature based on a peeling surface or exposed reinforcement. Hence, required properties are peeling surface and exposed reinforcement.

- Scaling - Scaling is a loss of surface mortar. It is listed in the inspection guidelines of Israel and Alberta. Condition rating is determined based on depth, type of exposed aggregate and if reinforcement steel is visible. No clear distinguishing feature is given between scaling and freezethaw.

- Abrasion / Wear - This defect is listed in Californian and Israeli guidelines. Both distinguish the condition states based on the kind of exposed aggregate. Israel also takes the reinforcement into account. Hence, the required properties are if coarse aggregate is visible and/or if reinforcement steel is exposed

\section{INTEGRATING INSPECTION INFORMATION INTO IFC}

Existing inspection guidelines are intended for being used by human inspectors. Little consideration is given on how the output of an inspection can be embedded into a structured, electronic model.

Guidelines only specify paper-based report templates as a documentation format. Sketches are to be made on separate paper. The advantage of this approach is its flexibility which at the same time poses the main research challenge: Transferring this to a well-structured model automatically reduces the flexibility. For example, an inspector can always make freehand notes or sketches on a paper form to document details beyond the predefined form structure. A well-structured electronic model, instead, 
341 only allows using it in a predefined manner. Hence, it is important to analyse and identify

342 documentation requirements so that the predefined model can fully represent these guideline

343 requirements.

344 We use IFC for several reasons to demonstrate this integration process. IFC is a neutral and open

345 exchange format that is widely spread in the industry and supported by most of the relevant software

346 packages. It is well documented and supports 3D building models. IFC requires a hierarchical data

347 structure and defines three basic components for modelling buildings: objects, relationships and

348 properties. Objects are abstract entities, structured in an ordered hierarchy. Instances of these entities

349 are used to represent a real life element or object. Relationships relate different objects to each other

350 and properties add context information to an object. Before starting to select the appropriate entities,

351 we need to restructure the inspection and defect information.

352 STRUCTURING THE DATA

353 Three data blocks are modelled: general information about the bridge, responsible people for the

354 inspection and background information to the inspection. The first block is meant for linking a report

355 to a specific bridge. It can be ignored as we are integrating the defect information directly into one

356 holistic model. The second block is general information about the inspection, more precisely:

357 inspection type, weather condition and time duration. And the third block is the inspecting person.

358 Additional people, such as an advisor, could be added likewise. Database forms could be used to

359 provide condition summaries automatically once the data is available in order to present an inspector a

360 familiar report format. More sophisticated ways of presenting the defect data could be developed,

361 such as filtering methods or deterioration over time, but this is out of scope of this paper. Figure 3

362 visualizes the general information objects and their relation.

363 Next, different defect levels have to be considered. Even though an inspector on site does not

364 distinguish between directly visible defects and their interpretation, this distinction is essential when

365 integrating the data. The reason for this can be explained by analysing Table 1: The problem of

366 identifying and assessing a defect can be illustrated by looking at cracks: A crack can be a defect on 
367 its own, but it is also present within three other defect classes (Damaged pre-stress, delamination,

368 thaumasite / freeze-thaw). Hence, we are using two separate defect levels to accommodate this

369 separation between identification and assessment, element defects and defects. An element defect is

370 solely determined by its visible and geometrical appearance and has no condition assessment itself. It

371 affects only one element and is not a combination of multiple defect types. This kind of defects is

372 referred to as an element defect. As this defect represents a unique defect appearance at a specific

373 time, a distinct instance is added for each visual element defect during each inspection. It is explicitly

374 related to one specific inspection. This way, by representing a defect appearance separately for each

375 inspection, the expansion of an element defect over time is documented.

376 To group these multiple element defect instances, a defect is introduced which can group element

377 defects timewise and type-wise. To give an example: corrosion can induce cracks, spalling, bleeding

378 or even invisible element defects such as delamination. All these element defects should be tied

379 together as they originate from the same source. Additionally, the deterioration over time should be

380 represented. Hence, each time an element defect is identified, it is modelled as a separate instance of

381 element defect, as explained earlier, and tied together as one defect. This second kind of defect

382 generally combines element defects with the same cause over time. A defect has no visual

383 representation itself as it is a combination of visual element defects. It is referred to as defect.

384 Contrary to element defects, these defects can propagate over several elements and contain different

385 element defect types and multiple element defect instances. The condition assessment is done on the

386 level of a defect, not an element defect. The reason is, that a condition assessment stands for the

387 impact on a structure, this structural impact can only be assessed based on taking all contributing facts

388 into account, which are the element defects along with structural considerations. As they are

389 independent from a specific inspection, they are not directly tied to one. They are only related to the

390 inspections through the element defects. Figure 4 presents the defect hierarchy in a diagram. With the

391 outline of the objects, we can now determine how to structure the properties. Table 3 serves as basis

392 but further structuring helps to simplify the property sets. It is the aim to have small and simple, well- 
393 defined property sets for each element defect type without redundancy regarding the defect types or

394 properties.

395 The properties can be separated into two groups, one that directly describes a defect feature and is

396 unique to a defect type and a second, more general one that depends on the location and orientation of

397 a defect. Location and orientation dependent properties are relevant for multiple defects and are

398 structured in a separate property set. Another repetitive property set is the one describing exposed

399 reinforcement along with corrosion. This is also separated in an own property set. All resulting

400 property sets are listed in Table 4. Defect imagery taken during an inspection mostly shows a tiny part

401 of a bridge in a high resolution, such that the defect is properly represented in an image.

402 Unfortunately, these images might show severe distortions and, because mostly taken as close-up, it is

403 difficult for an inspector to fully understand the exact position and orientation of a defect based on an

404 image and prone to any kind of optical illusion. It is preferable to relatively register a camera position

405 to an element with the correct location and orientation. This way, understanding the defect relative to

406 the element is simple and by back-projecting the image onto the surface, distortions are removed. This

407 process is illustrated in Figure 5.

\section{SELECTING IFC ENTITIES}

409 Having the data structures and relations in place, we can now start the modelling process. We assume

410 to have an as-is IFC model of at least Level of Detail 300 (LoD 300) as input model which can be

411 used to integrate inspection and defect information. In general, IFC has a large variety of rather

412 abstract, universal entities, such as IfcObject for any kind of object and quite specific ones, such as

413 IfcBoiler, for a closed, pressure-rated vessel in which water or other fluid is heated. Each entity comes

414 with a semantic definition of what it is meant for.

415 The difficulty is to make the right choice in picking an IFC entity. It would be formally correct to just

416 always use a universal entity such as IfcProxy. However, it is good practice to be as specific as

417 possible in order to have a semantically meaningful model representation. This means to decide on the

418 deepest entity in the hierarchy that complies with the given semantic description. The problem is that 
419 some of the entities might not completely fit or appear to be too abstract. The typical answer to this

420 question is to propose an extension to IFC. This, however, has key limitations: first, an extension has

421 to pass the verification process and then has to be embedded into existing software. This process is

422 very time-consuming and might fail as an extension might never be accepted. Secondly, IFC already

423 is an extensive and complex standard that was developed over many years, involving many parties.

424 Constantly extending this standard bears the risk that it becomes unmanageable at some point. Hence,

425 we contend that the existing standard should be preferred where possible.

426 An Information Delivery Manual (IDM) to specify the technical components, activities and

427 information exchanges and a Model View Definition (MVD) to specify the data exchange schema to

428 serve the IDM can be used for defining and, even more importantly, checking the conformity of any

429 given data exchange structure. This allows to specify the way the properties are represented in an IFC

430 file and which IFC entities are allowed to be used. Developing this is beyond the scope of this work,

431 but was done based on this work by Ma et al. (Ma et al. 2017).

\section{RESULTS}

\section{SELECTED IFC ENTITIES}

434 For general information, the IFC standard contains an entity named IfcTask, which is defined to be

435 used to "describe an activity" such as "operation related activities" (buildingSMART International

436 Ltd. 2017b). It inherits from IfcProcess and does not have a geometrical representation. Name, title

437 and company of an inspector is put into IfcTask.Name. Any comments, including the weather

438 condition, is stored as IfcTask.Description. The inspection level or type of inspection (e.g. safety,

439 general or principal) is stored in IfcTask.WorkMethod as it matches the IFC attribute definition of

440 describing the method of work used in carrying out a task. The time and duration is linked to a related

441 IfcTaskTime. Besides the actual date, time and duration of an inspection, this entity also allows to be

442 used for scheduling an inspection and to compare planned and required inspection time. The IfcTask

443 describing the context information is assigned to each element defect, but not the defect itself, using

444 IfcRelAssignsToProcess. This way complies with the optimal structure described in Section 4.1., 
except that a person is not modelled as an own entity but an existing attribute is used. Figure 6

446 illustrates how to use IfcTask to model inspection context information. For defects and their

447 properties, IfcElementAssembly is defined as representation of "complex element assemblies

448 aggregated from several elements, such as discrete elements, building elements, or other elements"

449 and "it does not need to have an explicit geometric representation" (buildingSMART International

450 Ltd. 2017b). Hence, it is appropriate for modelling a defect.

451 Besides grouping associated element defects, a defect also includes a condition rating, the actual

452 engineer's assessment. In IFC $2 \times 4$ a property set for condition rating was introduced: Pset_Condition.

453 This property set has three separate properties to describe a condition, namely AssessmentDate,

454 AssessmentCondition for an overall condition expressed in a short authority-dependent numerical

$455(1,2,3, \ldots)$ or alphanumerical $(\mathrm{a}, \mathrm{b}, \mathrm{c}, \ldots$ or good, fair, poor, $\ldots)$ unit and AssessmentDescription for a

456 qualitative, text-based description of the condition. The defect is assigned to an IFC element using the

457 aggregation relationship IfcRelAggregates. This way, a defect can be assigned to one or even multiple

458 elements in case a defect is propagating over several elements. Figure 7 demonstrates how to use the

459 presented IFC structure for modelling a defect.

460 IfcSurfaceFeature is used for modelling element defects. This entity is derived from IfcElement and

461 thus has an own geometrical representation. It is defined as "a modification at (onto, or into) ... the

462 surface of an element" and may minor increase, remain or decrease the mass of an element

463 (buildingSMART International Ltd. 2017b). A tessellated shape representation is modelled using

464 IfcShapeRepresentation. This gives flexibility about form and position of a defect in 3D. It consists of

465 a list of 3D points, defined in IFC as IfCCartesianPointList $3 D$, and based on this, corresponding

466 triangles defined by IfcTriangulatedFaceSet. Any other valid IFC shape representation would be

467 feasible. This one has the advantage of simplifying the subsequent texture mapping. Element defect

468 properties are added similarly as for defects using IfcPropertySets with the difference, that this time

469 there is no standard property set definition that can be referred to. The property sets are individually

470 created per defect class based on the individual properties from Table 4 . The last column indicates the

471 most suitable data type. In general, IFC allows to assign property sets to multiple IfcElements. 
472 However, in this case a property set describes individual defect measurements taken at a specific point

473 in time and thus must be assigned to the corresponding IfcSurfaceFeature exclusively which

474 represents the specific element defect from which measurements were taken. Figure 8 presents an

475 example crack modelled as element defect.

476 Having the triangles of the already modelled element defect, we can directly use IfcTextureVertexList

477 and IfcIndexedTriangleTextureMap to define a mapping of the 3D triangles to a $2 \mathrm{D}$ image. This

478 technique originates from Computer Graphics and is known as UV mapping (Murdock 2008). It is

479 illustrated in Figure 5. To each corner of a triangle in 3D, a corresponding set of 2D coordinates is

480 defined, whose coordinates are referred to as U and V (In the scope of IFC, these coordinates are

481 named $\mathrm{S}$ and $\mathrm{T})$. Texture coordinates $\mathrm{U}$ and $\mathrm{V}$ are specified in the range of $[0,1]$ and are scaled

482 automatically during runtime to the image size. It has to be mentioned that the use of the texture

483 mapping feature is not yet very well supported as, so far, IFC is mainly used during the design

484 process, where the benefit of having textured surfaces is significantly smaller. The texture image itself

485 is referenced as a uniform resource identifier (URI) by using IfcImageTexture. A URI can either be a

486 locally stored file or a resource located in a private or public network. The advantage of externally

487 referencing the texture file is that the IFC file is kept small and readable whilst binary code is outside.

488 A problem exists when an image texture file location changes or a resource in the network is no

489 longer available. This results in a corrupted IFC file. IfcBlobTexture offers a solution by embedding

490 the image binary data into an IFC file. This ensures the availability of the image data to the cost of a

491 significantly increased file size and complicates readability. Common image compression formats,

492 such as jpeg or png, are supported for both entities. This way, a surface texture can be assigned

493 explicitly to an element and it can even be precisely located on an element. Having this

494 transformation, we can directly take measurements in pixels from the image and convert them into

495 physical measurements in our 3D model space. The corresponding example IFC code is given in

496 Figure 9. 
498 In order to prove the feasibility of the presented concept, we defined a set of example defects,

499 modelled and augmented with potential real life data. We evaluated, which of the bridge inspection

500 requirements is supported by existing documentation formats (paper-based, image-based, dxf, existing

501 work based on IFC2x3, presented method). In addition, we implemented a prototype viewer and

502 evaluated it along with other most commonly used IFC viewers, to see, how much of the presented

503 scheme can be visualized and is supported by each viewer.

504 The fictitious exemplary inspection and defect situation for illustrating the concept was set as follows:

505 A simple example structure consists of two orthogonally oriented beams. They were inspected two

506 times, once in 2012 and once in 2014. During the inspection in 2012, a simple crack was detected

507 affecting beam one. It was oriented upwards starting at the bottom of one side of the element. In the

508 second inspection in 2014 this crack has increased in length and width and an inspector assumed that

509 it also structurally propagates to the other beam. In addition, a corrosion defect appeared on beam one

510 which was identified to be due to a spalling on two surface sides of the element including exposed and

511 corroding reinforcement. Pictures were taken from both defects. This situation was modelled using the

512 presented concept. A sketch of the defect location is presented in Figure 10.

513 We measured the performance by analysing which of the requirements is fulfilled by existing

514 documentation formats. Table 5 presents the results. The last column presents a performance score.

515 We tested the following attributes:

516 - General inspection information: Is there a way to model general inspection information as defined $517 \quad$ in Figure 3?

518 - Defect type: Is there a way to model defect types presented in Table 4 in a self-explanatory way?

519 - Defect location: Is there a way to exactly determine the location of the defect on the structure?

520 - Defect extent: Is it possible to extract the extent, absolutely and relatively to the structure?

521 - Defect severity: Is it possible to store a condition rating? 
- Defect cause: Can the cause of a defect be stored and explained, including an inspector's reasoning $523 \quad$ and conclusion?

- Additional defect properties: Is there a way to add properties to an element defect, depending on 525 the defect type and the properties listed in Table 4.

- 3D geometry: Is there a way to model arbitrary defect geometries in 3D?

- Group different defect types: If having a group of different element defect types, can they be linked such that their causal relationship is described?

- Group defects affecting multiple elements: If having a defect that propagates over multiple

530 elements, is there a way to group them such that their causal relationship is described

- Image registration / defect texturing: Can defect imagery be registered properly and placed on top of the element's geometry so that it is identically located and identically looking as it is on site?

- Machine readability: Can a computer extract, transform and process the stored data on a bridge inspection relevant level? As an example, this could mean to extract all cracks on beams from multiple models where crack width is greater than a given threshold.

- Easily shareable: Is there a way to send defect documentation electronically without taking much time or causing excessive costs?

- Fully integrated data type: Is the model and corresponding and referred documentation in one package or is it split in many chunks?

540 To compile the inspection building model files for the case study, we used a text editor and manually

541 added and manipulated the IFC files. This required several hours. It is obviously not the intended way

542 for eventual implementation, but in the absence of BIM tools that can model and export IFC files with

543 the proposed schema, it is necessary for the case study. In the authors' opinion, implementation in

544 existing BIM software can reduce the time required for integrating defect information into a BIM 
545 model at least to the time required to document inspection findings with existing methods, and likely

546 much more.

\section{VIEWER IMPLEMENTATION}

548 A prototypical viewer was implemented based on the Gygax research platform (Huethwohl et al.

549 2017). This platform is written in $\mathrm{CH}$ and $\mathrm{C}++$ and enables the use of typical data formats for AEC

550 industry (images, videos, point clouds, BIM models) and their preferred processing libraries in a

551 uniform way. Main supported data formats are images, videos, point clouds and BIM models. In the

552 scope of this work, the BIM functionality was particularly used. Gygax supports IFC files and utilizes

553 the IFC Engine DLL published by RDF (RDF 1td. 2017). This library supports reading and writing of

554 IFC files and also has a built-in geometry kernel which translates text-based IFC geometry description

555 into a triangle mesh that can be processed by 3D engines. Gygax uses the Helix Toolkit for

556 visualization of supported data types in one 3D space. More specifically, Helix Toolkit (Holance et al.

557 2016) is used in combination with SharpDX, which is a .NET wrapper of the DirectX API, to allow

558 high performance GPU processing even with large input datasets.

559 When opening an IFC file with embedded defect information, a two-columned window is presented.

560 One column is to visualize the 3D geometry of the model including the overlaid defect textures. On

561 zooming in to a specific defect, the defect gets clearly visible. Even small details, like cracks, can be

562 inspected. Defects that propagate over multiple surfaces of an element, are visualized on each

563 corresponding surface. Examples are given in Figure 11.

564 A second column is a tree view that maps the logical tree structure of the IFC model. When clicking

565 on a specific model element in the 3D view, the corresponding node in the tree gets highlighted.

566 Additional information, such as property sets, is presented as children nodes. Figure 12 shows this

567 tree. The first node represents the beam itself, followed by the defect crack, which has two children:

568 the element defect representation and a condition rating. The condition rating relates to the latest

569 available information, hence it states the last inspection date as assessment date. Within the element

570 defect, one can find the inspection information in the IfcProcess node, followed by the defect type 
571 dependent properties and the defect location properties. Each node lists the corresponding property

572 details.

573 As a final evaluation step, we tested the IFC viewer along with most commonly used IFC viewers to

574 see how much of the presented concept is supported by each viewer. The IFC viewers were chosen

575 from a review paper (Abanda et al. 2015) and additional commonly known viewers were added. The

576 performance of each viewer was examined by answering the following questions. Results are listed in 577 Table 6.

578 - Open file: Is it possible to open the file at all?

579 - Show 3D geometry: Are the two example beams correctly presented?

580 - Show element defect geometry: Is the geometry of each element defect visualized accurately?

581 - Show element defect texture: Is the texture of the element defect correctly shown? Is the

582 registration correct?

- Zoom in to see high resolution defect details: Is it possible to zoom in closely to see the details of

584 the crack and recognize the exposed reinforcement of the spalling?

- Show defect properties: Is a list shown with the defect condition properties?

- List nested element defects to defect: Is the relation between a defect and its corresponding element defects evident?

- List element defect properties: Are element defect properties listed including type dependent properties and location properties?

- General inspection information accessible: Is the corresponding general inspection information accessible along with the element defects?

\section{CONCLUSION AND FUTURE WORK}

593 We have presented results of a bridge inspection guideline analysis and a novel concept for

594 integrating RC defect information into an open and well-defined BIM model using the latest IFC 
standard (IFC 4 Add 2). In order to have a meaningful and significant basis on what information to

596 include into a BMS for the purposes stated, existing bridge inspection guidelines were examined and

597 requirements for defects and their properties were extracted, analysed and listed. We presented a

598 method on how to convert this information into an object-oriented hierarchy and how to assign

599 corresponding IFC 4 entities, both structurally and content-wise. The resulting IFC entities were

600 presented. A typical inspection situation was defined and findings were documented in an example

601 file in order to illustrate the feasibility of the concept. We developed a prototypical viewer which is

602 able to open and visualize the resulting model. The presented scheme was finally evaluated by

603 comparing it to common defect formats. It was shown that the existing IFC 4 standard is capable of

604 modelling bridge defects and general bridge inspection information in compliance with existing

605 bridge inspection guidelines. Limitations to this method exist in the extraction of defect properties, the

606 modelling approach in general and the IFC entity selection:

607 Presented defect properties are vaguely formulated as they have been extracted from existing

608 inspection guidelines, a clarification and consolidation of these guidelines is desirable. Using a

609 geometrical modelling approach require geometrical as-is IFC models for every bridge. These models

610 do not exist for a majority of bridges. Both research and authorities have identified this problem.

611 Research has started to develop methods to automatically model as-is bridges based on point cloud

612 data or images and authorities have started to require geometrical models for every newly built bridge.

613 Finally, the selection of an IFC entity to an object type, such as IfcSurfaceFeature for an element

614 defect, could be argued. There is no clear definition of which entity is correct, it is more a question of

615 interpreting the entities' description. Most important is the consistent use of entities to avoid

616 confusion in what the entities represent. We pointed out that an IDM/MVD is a possible solution to

617 this. Another limitation is the use of existing, most appropriate IFC entities which might not perfectly

618 fit. This directly raises the question of why not adding more distinct IFC entities, and using existing

619 ones. Our opinion is that the existing IFC standard should be used where possible as it already offers a

620 variety of different entities and excessive standard extensions lead to a confusingly complex standard.

621 Beyond that, proposing standard extensions is time consuming and they might not pass the validation 
process. It is expected, however, that the current IfcBridge standardization project will provide a

623 minimal set of extensions required to better capture the semantics of bridge elements. The project will

624 also develop MVDs for a number of use cases, including inspection, which will formally specify what

625 properties are required and how they must be represented in an IFC file. The MVD technology will

626 also allow to check instance models for compliance with these specifications.

627 Yet, inspectors can integrate their findings directly into a geometrical representation of a bridge with

628 the presented data format. Writing table-based reports is no longer required and reports can be

629 automatically extracted based on the model information. Resulting bridge models can easily be

630 exchanged across authorities or countries. Researchers can use this format to build bridge damage

631 datasets, which allow to assess and evaluate new inspection and maintenance methods. This will help

632 to improve the inspection and maintenance process and increase value for money for tax payers.

633 Integrating maintenance requirements and cost estimates is interesting for future work. This

634 information is usually given by an inspector during an inspection. Furthermore, although the

635 presented concept is able to cover and report the history of inspections and the corresponding defect

636 information thoroughly, it does not yet cover maintenance and repair work that was actually

637 conducted. Adding this information would help an inspector to fully understand a structure, its

638 degradation, conducted maintenance work and future serviceability.

\section{ACKNOWLEDGEMENTS}

640 We thank our colleague Amir Kedar from Kedmor Engineers Ltd. who provided insight and expertise

641 that greatly assisted this research.

\section{FUNDING}

643 This work is partly funded by Trimble Inc. and by the European Union's Seventh Framework

644 Programme for research, technological development and demonstration under grant agreement no.

645 31109806.0007. SeeBridge is co-funded by Funding Partners of the ERA-NET Plus Infravation and

646 the European Commission. The Funding Partners of the Infravation 2014 Call are: Ministerie van

647 Infrastructuur en Milieu, Rijkswaterstaat, Bundesministerium für Verkehr, Bau und Stadtentwicklung, 
648 Danish Road Directorate, Statens Vegvesen Vegdirektoratet, Trafikverket - Trv, Vegagerðin,

649 Ministere de L'ecologie, du Developpement Durable et de L'energie, Centro para el Desarrollo

650 Tecnologico Industrial, Anas S.P.A., Netivei Israel - National Transport Infrastructure Company Ltd.

651 and Federal Highway Administration USDOT.

\section{REFERENCES}

653 AASHTO. (2013). Manual for Bridge Element Inspection.

654 Abanda, F. H., Vidalakis, C., Oti, A. H., and Tah, J. H. M. (2015). “A critical analysis of Building 655 Information Modelling systems used in construction projects." Advances in Engineering $656 \quad$ Software, 90, 183-201.

657 Adhikari, R. S., Moselhi, O., and Bagchi, A. (2014). "Image-based retrieval of concrete crack 658 properties for bridge inspection." Automation in Construction, 39, 180-194.

659 Alberta Infrastucture and Transportation. (2008). Bridge Inspection \& Maintenance System.

660 Anil, E., Akinci, B., Garrett, J., and Kurc, O. (2013). "Representation of Damage Information for 661 Post-Earthquake Damage Assessment of Reinforced Concrete Frames." Computing in Civil 662 Engineering (2013), 825-832.

663 ASCE. (2017). “2017 Infrastructure Report Card - Bridges.”

$664<\mathrm{https}: / /$ www.infrastructurereportcard.org/cat-item/bridges/> (Sept. 1, 2017)

665 Badrinarayanan, V., Kendall, A., and Cipolla, R. (2015). "SegNet: A Deep Convolutional Encoder666 Decoder Architecture for Image Segmentation.”

667 Borrmann, A., Lukas, K., Zintel, M., and Schieß1, P. (2012). "BIM-based life-cycle management for 668 reinforced concrete buildings." International Journal of 3-D Information Modeling, 1-24.

669 buildingSMART International Ltd. (2017a). "Infrastructure." <http://www.buildingsmart670 tech.org/infrastructure $>$ (Apr. 6, 2017).

671 buildingSMART International Ltd. (2017b). "IFC Releases." <http://www.buildingsmart-tech.org/> 
673 Dekker, R. (2006). "The importance of having data-sets. The importance of having data-sets." $674 \quad$ Proceedings of the IATUL Conferences The.

675

676

Department for Transport and Highways Agency. (2014). Maintaining Strategic Infrastructure: Roads.

Eastman, C. M., Teicholz, P., Sacks, R., and Liston, K. (2011). BIM handbook: A guide to building information modeling for owners, managers, designers, engineers and contractors. John Wiley \& Sons.

Federal Highway Administration. (2003). Distress Identification Manual.

Hartmann, J. L., and Director, P. E. (2016). Bridge Information Model Standardization VOLUME II: SCHEMA ANALYSIS.

Highways Agency. (2007). Inspection manual for highway structures. TSO.

Holance, Objorke, Ikeough, and Iluvata82. (2016). "Helix Toolkit - 3D toolkit for .NET." $<$ https://github.com/helix-toolkit/helix-toolkit> (Sept. 1, 2017).

Huethwohl, P., Armeni, I., Fathi, H., and Brilakis, I. (2017). “Gygax Construction IT research platform for 2D \& 3D." <https://github.com/ph463/Gygax> (Sept. 7, 2017).

Johnson, M. B., Casey, W., O’ Donnell, L., Allec, P., Marshall, A. R., and Soden, D. (2016). Caltrans Bridge Element Inspection Manual.

Kedar, A., Peretz, S., Stuchevsky, D., Fathi, H., Lu, R., Vela, P., Singer, D., Sacks, R., Ma, L., Kattel, U., Brilakis, I., Huethwohl, P., Borrmann, A., and Daum, S. (2016). “Automated Compilation of Semantically Rich BIM Models of Bridges - Information Delivery Manual.”

Kong, J. S., Asce, M., Frangopol, D. M., and Asce, F. (2003). “Life-Cycle Reliability-Based Maintenance Cost Optimization of Deteriorating Structures with Emphasis on Bridges.”

Kouridi, C., and Brilakis, I. (2016). "User Requirements for Augmented Reality-based Bridge 
697 Lee, J. H., Lee, J. M., Kim, H. J., and Moon, Y. S. (2008). "Machine Vision System for Automatic 698 Inspection of Bridges.” 2008 Congress on Image and Signal Processing, IEEE, 363-366.

699 Ma, L., Sacks, R., and Zeibak-Shini, R. (2015). "Information modeling of earthquake-damaged $700 \quad$ reinforced concrete structures." Advanced Engineering Informatics, 29(3), 396-407.

701 Ma, L., Yosef, R., Muhic, S., Sacks, R., Kedar, A., Borrmann, A., Daum, S., Barutcu, B., Brilakis, I., 702 Liebich, T., and Huethwohl, P. (2017). “SeeBridge Next Generation Bridge Inspection:

703 Overview, Information Delivery Manual and Model View Definition," submitted to Automation 704 in Construction.

705 McGuire, B., Atadero, R., Clevenger, C., and Ozbek, M. (2016). "Bridge Information Modeling for $706 \quad$ Inspection and Evaluation.” Journal of Bridge Engineering, 21(4).

707 Minnesota Department of Transportation. (2003). Mn/DOT Distress Identification Manual.

708 Mirzaei, Z., Adey, B. T., Klatter, L., and Kong, J. S. (2012). The IABMAS Bridge Management 709 Committee Overview of Existing Bridge Management Systems.

710 Moore, M., Phares, B., Graybea, B., Rolander, D., and Washer, G. (2001). "Reliability of Visual 711 Inspection for Highway Bridges, Volume I: Final Report and Volume Ii: Appendices.” Baystate Roads Program Local Technical Assistance Program - Tech Note \#31, I(June), 1-4.

713 Murdock, K. (2008). 3ds Max 2009 bible. Wiley.

714 Phares, B. M., Washer, G. A., Rolander, D. D., Graybeal, B. A., and Moore, M. (2004). "Routine 715 Highway Bridge Inspection Condition Documentation Accuracy and Reliability." Journal of $716 \quad$ Bridge Engineering, ASCE, 9(4), 403-413.

717 Queensland Department of Main Roads. (2004). Bridge Inspection Manual.

718 RDF 1td. (2017). "IFC Engine DLL." < http://rdf.bg/ifc-engine-dll.html> (Feb. 15, 2017).

719 Sacks, R., Kedar, A., Borrmann, A., Ma, L., Singer, D., and Kattel, U. (2016). "SeeBridge 
721 Tanaka, F., Hori, M., Onosato, M., Date, H., and Kanai, S. (2016). "Bridge Information Model Based 722 on IFC Standards and Web Content Providing System for Supporting an Inspection Process.” 16th International Conference on Computing in Civil and Building Engineering, 188.

724 U.S. Department of Transportation. (2012). Bridge Inspector's Reference Manual.

725 Wallis, J. (2007). "Visual Data Collection for UKPMS.” The UKPMS User Manual.

726 William East, E., Nisbet, N., and Liebich, T. (2013). "Facility Management Handover Model View." $727 \quad$ Journal of Computing in Civil Engineering, 27(1), 61-67.

728 Zhu, Z., German, S., and Brilakis, I. (2011). "Visual retrieval of concrete crack properties for 729 automated post-earthquake structural safety evaluation.” Automation in Construction, 20(7), $730 \quad 874-883$. 